\title{
SUR LA FAUNE DES TRÉMATODES \\ DES OISEAUX DE TRANSBAÏKALIE
}

\author{
Par le professeur K.-I. SKRIABINE
}

Pendant l'été 1923, d'accord avec le Département d'agriculture de l'Orient, j'ai organisé la onzième expédition helminthologique russe en Transbaïkalie. Pendant un mois seulement, il a été possible de récolter des vers parasites dans ce lointain et intéressant pays, dont la faune helminthologique est encore peu connue.

Les membres de cette expédition étaient: le médecin-vétérinaire G.-G. Vitenberg, le médecin B.-P. Podjapolskaya et l'étudiant N.-P. Orloff, qui ont recueilli, entre autres matériaux, des trématodes de mammifères, d'oiseaux, d'amphibiens et de poissons.

Le but de cette note est de donner une, description sommaire des trématodes récoltés par l'expédition, chez les oiseaux de Transbaïkalie.

L'expédition a examiné 126 exemplaires d'oiseaux, qui se rapportent à 41 espèces. Chez 23 exemplaires, on trouve des trématodes, soit presque 18 pour cent d'oiseaux infectés. L'énumération des oiseaux, chez lesquels furent trouvés les trématodes, est donnée dans le tableau ci-contre.

Les oiseaux étaient parasités par 12 espèces de trématodes qui se rapportent aux 5 familles suivantes: Lepodermatidæ, Opisthorchidæ, Notocotylidæ, Echinostomidx et Holostomidæ.

Dans le matériel étudié, une espèce nouvelle du genre Plagiorchis Lühe fut découverte; je la dédie au voyageur sibérien très connu, G.-N. Potanin : Plagiorchis potanini n. sp. J'ai considéré en outre un parasite d'Anas boschas L. comme une variété nouvelle de Plagiorchis maculosus (Rud.) var. anatis $\mathrm{n}$. var. Les autres trématodes se rapportent à des espèces bien étudiées, appartenant à la faune de l'Europe.

\section{A. Lepodermatidæ}

Deux genres de cette famille : Plagiorchis Lühe et Prosthogonimus Lühe, sont seuls représentés dans le matériel récolté par l'expédition.

Annales de Parasitologie, T. VI, $\mathrm{N}^{\circ} 1,-1^{\mathrm{er}}$ janvier 1928 , p. 80-87. 


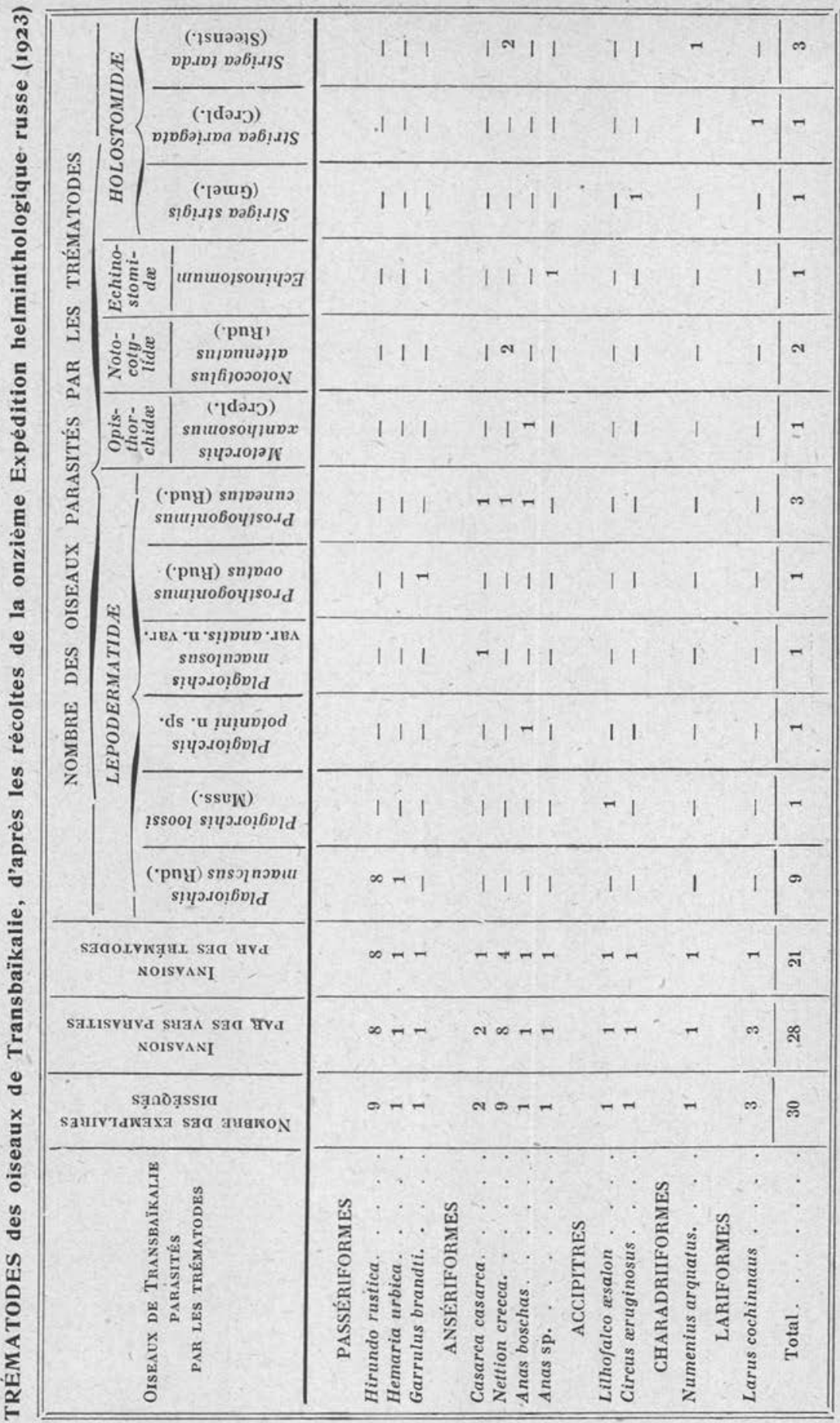

AnNales de Parasitologie, T. VI, No $1,-1$ er janvier 1928. 


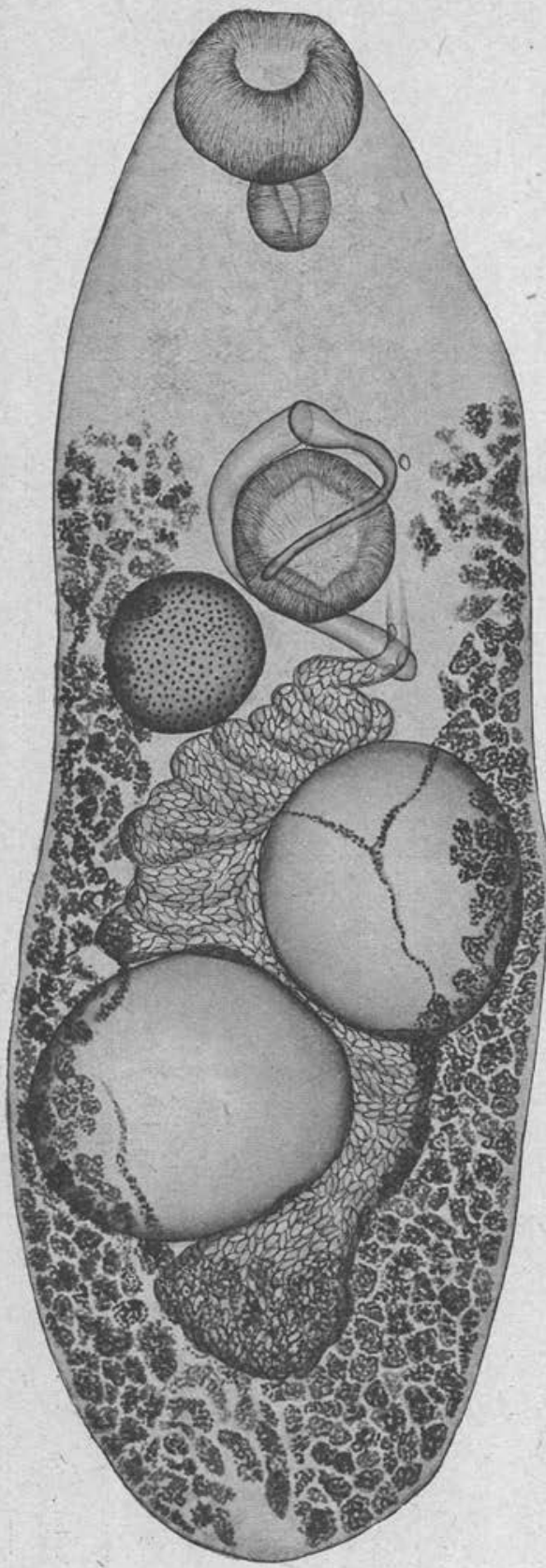

Fig. 1. - Plagiorchis maculosus (Rud.), var, anatis nov. var.

\section{Plagiorchis Lühe $\mathbf{1 8 9 9}$}

- B.-G. Massino publiera bientôt une monographie du genre Plagiorchis avec la description d'espèces nouvelles.

Dans le-matériel récolté, il y a quatre représentants de ce genre.

\section{Plagiorchis maculosus (Rud., 1902)}

Cette espèce fut trouvée chez Hirundo rustica 8 fois sur 9 exemplaires disséqués et chez Hemaria urbica, une fois chez un seul exemplaire disséqué. Les parasites étaient logés pour la plupart dans l'intestin et par exception, dans le rectum. Le nombre des exemplaires trouvés variait entre 1 et 30 chez chaque oiseau. Le parasite est typique et ne diffère par aucun détail de son organisation des formes européennes.

2. Plagiorchis maculosus (Rud.), var. anatis, nov. var.

A la dissection d'un Casarca casarca, tué le 28 août 1923 , on trouva dans l'intestin un exemplaire que nous devons considérer comme une nouvelle variété de P. maculosus (Rud.) (fig. 1). Cette variété s'est probablement formée sous l'influence de l'appropriation à un nouvel hôte : l'espèce typique habite, comme on le sait, dans l'intestin des passereaux, le plus souvent chez les hirondelles, et la var. anatis est adaptée à un Anatidæ, Casarca casarca. 
Description. - Le corps a $2 \mathrm{~mm}$., 2 de long et $0 \mathrm{~mm}$., 75 de largeur maxima dans la région des testicules. Ventouse buccale : $0 \mathrm{~mm}$., $23 \times 0 \mathrm{~mm}$., 25 ; pharynx : $0 \mathrm{~mm}$., $09 \times 0 \mathrm{~mm}$., 09 . Ventouse ventrale : $0 \mathrm{~mm}$., 25 de diamètre. Le centre de la ventouse ventrale est situé à la distance de $0 \mathrm{~mm}$., 75 de l'extrémité céphalique. Les testicules mesurent $0 \mathrm{~mm} ., 4 \times 0 \mathrm{~mm}$., 4 et se touchent l'un l'autre. L'ovaire est situé immédiatement en arrière et à gauche de la ventouse ventrale et mesure $0 \mathrm{~mm}$., $22 \times 0 \mathrm{~mm}$., 24 .

Les glandes vitellogènes consistent en petits follicules commençant au niveau de l'orifice génital, 'c'est-à-dire immédiatement en avant du bord antérieur de la ventouse ventrale et finissant dans la partie postérieure du corps. Les glandes vitellogènes du côté droit ne sont nulle part en contact avec celles du côté gauche. L'utérus est massé en grande partie en arrière des testicules, et passe entre l'ovaire et le testicule antérieur. Les œufs atteignent $0 \mathrm{~mm}$., 036 de long sur $0 \mathrm{~mm}$., 022 de large. La poche du cirre est courbée en arc ; son extrémité postérieure atteint le niveau du bord postérieur de l'ovaire, n'arrivant pas cependant jusqu'au bord antérieur du testicule antérieur. Une différence caractéristique entre cette variété et le $P$. maculosus typique, consiste dans la structure des glandes vitellogènes : chez la variété anatis, les follicules sont assez petits et forment une couche si épaisse, qu'elle donne l'impression d'une masse tout à fait compacte, tandis que chez le $P$. maculosus typique des hirondelles, les follicules sont plus gros, plus grands et sont répartis sur une couche moins épaisse. Je trouve cette différence assez importante car il est nécessaire maintenant de porter son attention sur la structure des glandes vitellogènes, à cause de leur signification dans la systématique (1).

\section{Plagiorchis loossi Mass., 1926}

Dans l'intestin de Lithofalco æsalon a été découvert un trématode identique au Plagiorchis loossi, que Massino vient de décrire dans sa monographie, et qui fut trouvé dans l'intestin de Lanius collurio (Département du Don). Cette espèce diffère de tous les autres Plagiorchis par la dimension de l'ovaire, plus grand que les testicules.

\section{Plagiorchis potanini n. sp.}

Cette espèce (fig. 2), fut rencontrée dans l'intestin d'un canard domestique Anas boschas L., disséqué le 6 septembre 1923. Il en a été récolté 23 exemplaires.

(1) Consulter à ce sujet l'ouvrage de Vitenberg sur les Cyclocœlidæ. 
Description. - Le corps atteint $1 \mathrm{~mm} .,-77$ de long sur $0 \mathrm{~mm} ., 47$ de large au niveau des testicules. La ventouse buccale mesure

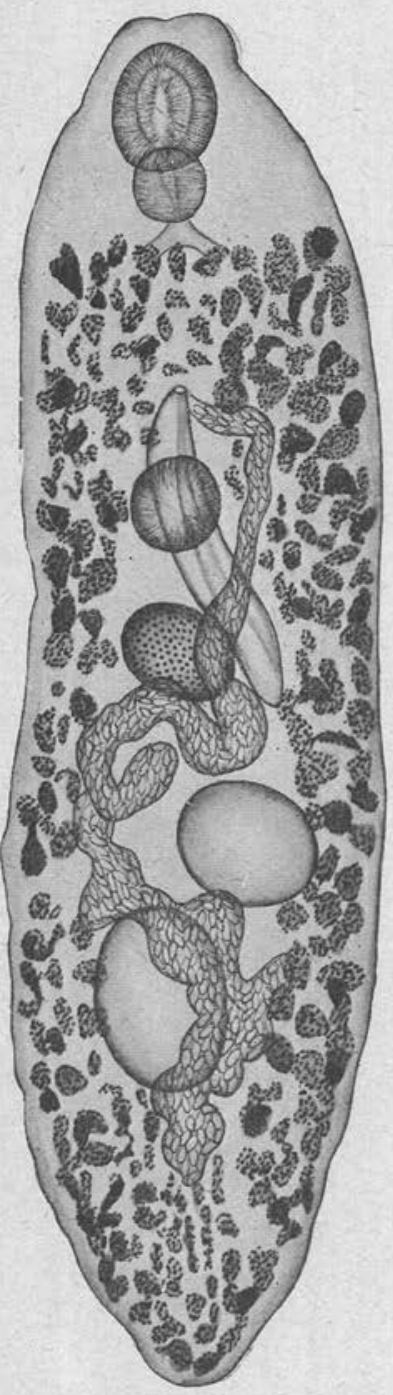

Fig. 2.- Plagiorchis potanini $\mathrm{n}$. sp.

0 mm., $158 \times 0$ mm., 136. Pharynx très robuste; ventouse buccale atteignant $0 \mathrm{~mm} ., 1$ de long sur $0 \mathrm{~mm}$., 1 de large. La ventouse ventrale a $0 \mathrm{~mm} ., 136$ de diamètre ; son centre se trouve à $0 \mathrm{~mm}$., 63 de l'extrémité céphalique.

Les testicules de 0 millimètre, $18 \times 0 \mathrm{~mm}$., 18 sont arrondis et situés l'un derrière l'autre. Entre les deux testicules et entre le testicule antérieure et l'ovaire, se trouvent les anses de l'utérus. L'ovaire est ovale, à grand axe transversal, il mesure $0 \mathrm{~mm}$., $11 \times 0 \mathrm{~mm}$., 136 et se trouve un peu en arrière de la ventouse ventrale, à une distance de $0 \mathrm{~mm}$., 06. Les glandes vitellogènes commencent près du pharynx et atteignent l'extrémité postérieure; elles se composent de follicules épais, confluant sur la ligne médiane, à la partie antérieure du corps et à la partie postérieure.

L'utérus occupe la portion médiane du ver, ne traversant pas la ligne postérieure du testicule postérieur. L'orifice génital se trouve à une distance de $0 \mathrm{~mm}$., $07 \mathrm{du}$ bord antérieur de la ventouse ventrale. Les œufs mesurent $0 \mathrm{~mm}$., $032 \times 0 \mathrm{~mm}$., 018 . Une très longue poche du cirre, longue de $0 \mathrm{~mm}$., 454 dépasse en arrière la limite postérieure de l'ovaire.

Cette espèce est proche du Plagiorchis triangularis (Dies., 1850), de Merops apiaster, auquel elle ressemble par la topographie des glandes vitellogènes et de la poche du cirre, très développée, qui traverse le bord postérieur de l'ovaire; mais cependant notre 
espèce diffère visiblement du $P$. triangularis par la dimension de son pharynx, les dimensions des ventouses ventrale et buccale, les dimensions de l'ovaire et des testicules. La poche du cirre de notre espèce est presque droite tandis que chez le Plagiorchis triangularis, elle se courbe en cercle, et atteint une plus grande largeur ; en ligne droite, elle occuperait presque la moitié du corps.

\section{Prosthogonimus Lühe 1899}

Ce genre fait partie de l'étude monographique que j'ai publiée avec W.-P. Baskakowy. Nous avons groupé les espèces de ce genre en sous-genres, ayant pris pour principe de la division le degré du développement des organes génitaux. Dans la bourse de Fabricius des biseaux de Transbaïkalie, on a constaté deux espèces de ce genre:

\section{Prosthogonimus (Ultragenotrema) ovatus (Rud., 1803)}

Un exemplaire de cette espèce fut recueilli dans la bourse de Fabricius de Garrulus brandti, tué aux environs de Chita, le 9 octobre 1923. Aucun ver parasité n'avait été constaté chez cet oiseau jusqu’à présent. Cet oiseau doit donc être considéré comme un hôte nouveau de ce trématode.

\section{Prosthogonimus (Macrogenotrema) cuneatus (Rud., 1809)}

Ce parasite a été trouvé dans la bourse de Fabricius de Nettion crecca et de Casarca casarca. Ces deux oiseaux sont de nouveaux hôtes pour ce Prosthogonimus. Il est évident que, d'après l'étude monographique du genre Prosthogonimus, il faudra diviser l'espèce $P$. cuneatus en plusieurs sous-espèces et il faudra rapporter les parasites trouvés chez les oiseaux de Transbaïkalie, décrits plus haut, à une sous-espèce particulière.

\section{B. Opisthorchidæ}

\section{Metorchis}

\section{Metorchis xanthosomus (Crepl.)}

Ce parasite également propre à la faune européenne, fut trouvé en deux exemplaires dans la vésicule biliaire du canard domestique: Anas boschas, L., tué le 6 octobre aux environs de la Chita. 


\section{Notocotylidæ}

IV. Notocotylus

\section{Notocotylus attenuatus (Rud.)}

Ce parasite, également propre à la faune de l'Europe, fut trouvé chez deux sarcelles, Nettion crecca, sur neuf étudiées. Chez une sarcelle-il y avait 106 exemplaires dans le cæcum ; chez une autre 11 exemplaires.

\section{Echinostomidæ \\ V. Echinostomum Rud. \\ 9. Echinostomum sp.}

Ce trématode fut trouvé une fois dans l'intestin d'un canard, Anas sp. A cause du petit nombre des exemplaires, il fut impossible d'en déterminer l'espèce.

\section{E. Holostomidæ}

VI. Strigea Abildg.

Le genre Strigea est représenté par 3 espèces, trouvées chez 4 hôtes.

\section{Strigea strigis (Gmelin)}

Cette espèce, commune chez les oiseaux de proie de la faune de l'Europe, fut recueillie au nombre de deux exemplaires, dans l'intestin du busard des marais, Circus æruginosus, tué le 3 octobre 1923 aux environs de la Chita.

\section{Strigea variegata (Crepl.)}

Cette espèce, fréquente chez les mouettes d'Europe, fut trouvée dans l'intestin de Larus conchinnans, tué le 17 octobre 1923 sur le lac Kinon. Il en existait 28 exemplaires.

\section{Strigea tarda (Steenstr.)}

Cette espèce, peu étudiée, fut trouvée chez 2 hòtes: Nettion crecca et Numenius arquatus. Sur 9 sarcelles disséquées, le para- 
site fut trouvé deux fois. Chaque fois, on en récolta 12 exemplaires. Chez Numenius le nombre des exemplaires était de 30.

En plus de ces 12 espèces de trématodes, la onzième expédition a constaté la présence d'un Plagiorchis sp. chez Passer domesticus et d'un Notocotylus sp. chez Machetes pugnax. Ces parasites ont.été égarés en route et n'avaient pu être examinés que sommairement auparavant.

Le présent travail mentionne 12 espèces de trématodes trouvés chez 11 espèces d'oiseaux de Transbaïkalie. C'est le premier d'une série de travaux sur l'étude de la faune helminthologique de la Sibérie. Cette étude envisagera, en des publications ultérieures, les vers parasites des mammifères, des amphibiens et des poissons de Transbaïkalie, et il est à souhaiter que le matériel, récolté en abondance, réserve d'intéressantes découvertes aux spécialistes qui l'étudient.

Division helminthologique de l'Institut vétérinaire expérimental de l'Etat (Moscou). 